\title{
Optimal energy management for hybrid-electric aircraft
}

\author{
Fosé Pedro Soares Pinto Leite \\ Faculty of Aerospace Engineering, Technische Universiteit, Delft, The Netherlands, and \\ Mark Voskuijl \\ Faculty of Military Sciences, Netherlands Defence Academy, Den Helder, The Netherlands
}

\begin{abstract}
Purpose - In recent years, increased awareness on global warming effects led to a renewed interest in all kinds of green technologies. Among them, some attention has been devoted to hybrid-electric aircraft - aircraft where the propulsion system contains power systems driven by electricity and power systems driven by hydrocarbon-based fuel. Examples of these systems include electric motors and gas turbines, respectively. Despite the fact that several research groups have tried to design such aircraft, in a way, it can actually save fuel with respect to conventional designs, the results hardly approach the required fuel savings to justify a new design. One possible path to improve these designs is to optimize the onboard energy management, in other words, when to use fuel and when to use stored electricity during a mission. The purpose of this paper is to address the topic of energy management applied to hybrid-electric aircraft, including its relevance for the conceptual design of aircraft and present a practical example of optimal energy management.

Design/methodology/approach - To address this problem the dynamic programming (DP) method for optimal control problems was used and, together with an aircraft performance model, an optimal energy management was obtained for a given aircraft flying a given trajectory.

Findings - The results show how the energy onboard a hybrid fuel-battery aircraft can be optimally managed during the mission. The optimal results were compared with non-optimal result, and small differences were found. A large sensitivity of the results to the battery charging efficiency was also found.

Originality/value - The novelty of this work comes from the application of DP for energy management to a variable weight system which includes energy recovery via a propeller.
\end{abstract}

Keywords Dynamic programming, Optimal control, Flight performance, Hybrid aircraft, Optimal energy management

Paper type Research paper

\section{Nomenclature}

\section{Symbols}

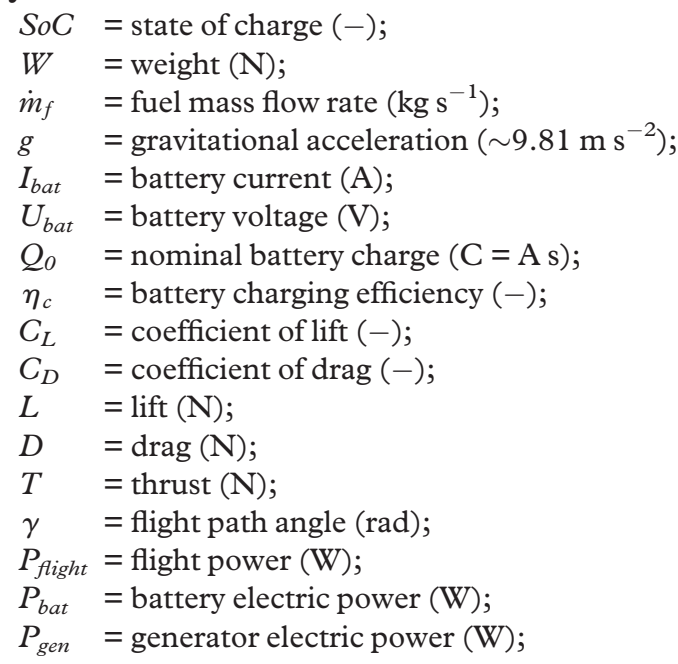

The current issue and full text archive of this journal is available on Emerald Insight at: https://www.emerald.com/insight/1748-8842.htm

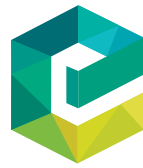

Aircraft Engineering and Aerospace Technology 92/6 (2020) 851-861

Emerald Publishing Limited [ISSN 1748-8842] [DOI 10.1108/AEAT-03-2019-0046]
$P_{I C} \quad=$ IC engine shaft power (W);

$P_{I C_{\max }}=$ IC engine max. shaft power (W);

$P_{\text {inv }}=$ motor inverters DC power $(\mathrm{W})$;

$P_{\text {max }}=$ max.rotor $\operatorname{power}(\mathrm{W})$;

$\eta_{\text {prop }}=$ propeller efficiency $(-)$;

$\eta_{G B}=$ gear box efficiency $(-)$;

$\eta_{M}=$ motor efficiency $(-)$;

$\eta_{\text {inv }}=$ invertors efficiency $(-)$;

$\eta_{\text {gen }}=$ generator efficiency $(-)$;

$\Gamma_{f} \quad=$ generator throttle setting $(-)$;

$S F C=$ specific fuel consumption $\left(\mathrm{kg} \mathrm{W}^{-1} \mathrm{~s}^{-1}\right)$;

$\rho \quad=$ air density $\left(\mathrm{kg} \mathrm{m}^{-3}\right)$;

$A \quad=$ rotor area $\left(\mathrm{m}^{2}\right)$;

$V \quad=$ true air speed $\left(\mathrm{m} \mathrm{s}^{-1}\right)$; and

$C_{P} \quad=$ rotor power coefficient $(-)$.

(C) José Pedro Soares Pinto Leite and Mark Voskuijl. Published by Emerald Publishing Limited.This article is published under the Creative Commons Attribution (CC BY 4.0) licence. Anyone may reproduce, distribute, translate and create derivative works of this article (for both commercial and non-commercial purposes), subject to full attribution to the original publication and authors. The full terms of this licence may be seen at http://creativecommons.org/licences/by/4.0/legalcode

Received 9 March 2019

Revised 17 November 2019

Accepted 25 March 2020 


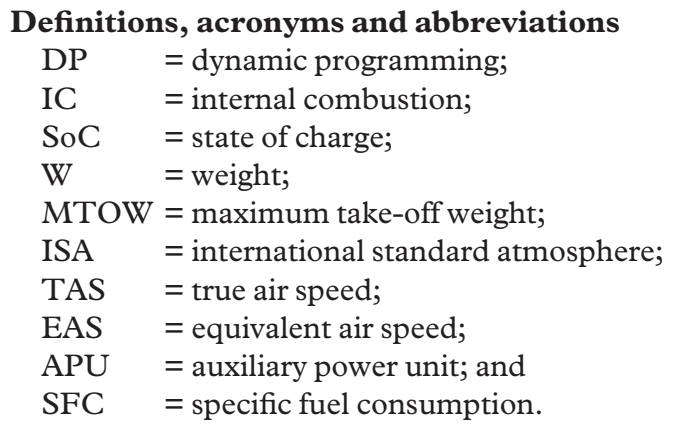

\section{Introduction}

In the past decades, the trend towards "More Electric Aircraft" has materialized in new airliners such as the Boeing 787. This trend is powered by the high reliability and low maintenance requirements of modern mechatronic systems (Rosero et al., 2007; Sarlioglu and Morris, 2015; Mavris et al., 2010) and, so far, has targeted only non-propulsive systems such as the ice protection system. One possible future evolution for this trend is electric propulsion: the use of electricity available onboard to power the propulsion system. This opens new possibilities for aircraft design, such as distributed electric propulsion or radically new aircraft configurations (Sehra and Whitlow, 2004; Gohardani et al., 2011). This trend maybe accelerated in the near future by several factors: higher oil prices, carbon taxes, changes in politics, etc.

Several research groups around the world are doing design exercises on hybrid aircraft with different configurations (Pornet et al., 2013; Pornet and Isikveren, 2015; Harmon et al., 2006; Voskuijl et al., 2017). Unfortunately, the results of these exercises indicate only marginal improvements in fuel efficiency when hybrid electric designs are compared against conventional propulsion designs. Besides, these marginal improvements are conditionally dependent on the future evolution of battery energy density. To improve these results, it has been proposed to integrate onboard energy management in the aircraft design methodology (Perullo and Mavris, 2014).

In the context of hybrid vehicles, onboard energy management is defined as the management of the different energy sources available onboard during a mission (Serrao et al., 2011). In other words, it is the selection of the rate of energy consumption from each available source at each moment. This management can be done in real-time or predefined before a mission, depending on the selected control methodology. When optimization techniques are used to optimize the onboard energy management, it becomes optimal and can be referred to as optimal energy management.

When integrated in the aircraft design methodology, the optimal energy management is used in each step of the aircraft sizing loop, minimizing the block fuel for the entire mission. This will lead, owing to synergies, to a fuel efficiency improvement of the final hybrid aircraft design, potentially making hybrid aircraft a more attractive option to decrease fuel consumption and emissions in aviation (Perullo and Mavris,
2014; Perullo et al., 2014; Ficarella et al., 2018; Donateo et al., 2018b, 2019).

Despite the theoretical advantages, the integration of onboard energy management in the design exercise is not yet in wide spread use. Most of the design exercises involving hybrid aircraft published so far fix, a priori, the power split between the fuel power and electric power and not changing these values during the optimization run. A notable exception is the work of Donateo et al. (2018b), where a rule-based controller regulating the power split is active during the simulation. The conclusions of supra point that "[...], more advanced energy management strategies need to be developed." In a second research study by the same authors (Donateo et al., 2018a), dynamic programming (DP) algorithms were used to optimize the energy management of an aircraft in an approach very similar to the one in the present work, although with relevant differences (e.g. the effect of variable weight in performance was not taken into account).

In this work, no attempt will be made in integrating optimal energy management in the design exercise. Instead, another step towards this goal is taken: an improved method to determine the optimal energy management for hybrid aircraft is presented and studied. Similar methods were presented in previous works (Bradley et al., 2009; Bongermino et al., 2017; Donateo et al., 2018a), but the models used in these studies include neither the effect of weight reduction during the mission nor energy recovery. The weight reduction detail in particular becomes very relevant when considering long missions (Hepperle, 2012). The present work will also attempt to investigate which are the main factors influencing optimal energy management for a fixed-wing general aviation aircraft.

Besides directly improving the fuel economy of a hybrid aircraft during preliminary design, at least one more reason exists to study optimal energy management in this context: the development of non-optimal controllers for use in operational environments. For most real-life problems, the deployment of global-optimum real-time controllers is simply not possible. This is an effect of several characteristics of optimal controllers (e.g. the associated computational burden and the lack of robustness to certain types of external disturbances) that limit their use in operational context. To avoid these limitations,

Figure 1 Hybrid electric architectures

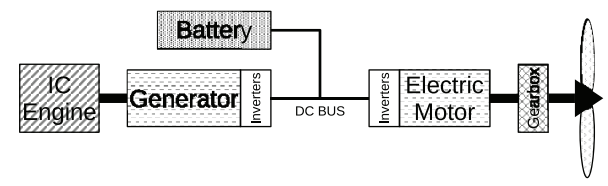

(a)

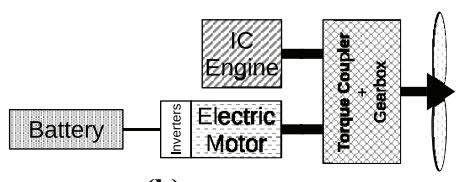

(b)

Notes: (a) Serial; (b) parallel; bold lines: mechanical link, solid lines: electrical link 
non-optimal controllers are usually developed and tuned by comparing their performance against optimal controllers in high-fidelity simulation environments (Guzzella and Sciarretta, 2013; Sinoquet et al., 2009; Liu and Peng, 2008). This application of optimal energy management will be briefly addressed in the present work, as the presented methodology can also be promptly used for this purpose. Some remarks regarding the application of optimal controllers to hybrid aircraft will also be given.

Relevant hybrid aircraft technologies, the optimal control problem, the aircraft under study and the mission will be described in the first half of this paper. In the second half, results will be presented and discussed, and conclusions will be drawn.

\section{Background}

Hybrid aircraft, unlike hybrid cars, are a relatively new topic, and more research needs to be done to make them a viable and efficient mean of transportation. However, some promising steps are being taken: companies specialized in small/general aviation aircraft developed or are developing fully electric aircraft and hybrid electric aircraft (Brelje and Martins, 2018). Some of the technologies developed for general aviation will, hopefully, be exported to airliners, the largest segment of the market.

Despite all the hopes surrounding hybrid aircraft, they face some major difficulties in the fight for market share. The most important one is likely to be the enormous cost of new technology development and certification, very difficult to overcome, as the expected gains in fuel efficiency are quite small. Aircraft manufacturers are not able to finance the development cost of a new aircraft unless it delivers substantial reductions in fuel consumption.

In this section, some background remarks regarding hybrid vehicle technology and hybrid aircraft technology will be given. The aircraft used as a case study during the present work will also be described. The topic of design methods for hybrid vehicles, although relevant, is not going to be addressed, as it is outside of the scope of this work, and interested readers are referred to specialized literature (Silvas et al., 2016). This section will also look into the problem of energy management for hybrid aircraft and how to address its optimization for hybrid vehicles in general and hybrid aircraft in particular.

\section{Types of architectures for hybrid electric vehicles}

Despite not being the main topic of this work, it is important to give the reader some insights on the two main hybrid electric architectures used for propulsion purposes: the serial architecture and the parallel architecture (Guzzella and Sciarretta, 2013; Wall and Meyer, 2017). Both are illustrated in Figure 1, where the application is a fixed-wing aircraft powertrain.
The main difference between both is the way the electric and the fuel-powered systems are connected: in the parallel case, this is done using a mechanical coupling mechanism (e.g. a gearbox), while in the serial case, the connection is done through a central electrical bus. In the latter case, the fuelpowered system (e.g. an internal combustion [IC] engine) is connected to a generator to provide energy to the bus.

Both architectures have pros and cons: in the parallel architecture, complicated mechanical coupling mechanisms must be designed and maintained, while in the serial architecture, the complexity is in the design of a large and redundant electrical system. The choice between architectures is usually made very early in the design phase, and for small aircraft, the serial architecture is usually favoured to take advantage of the high-specific power of electric motors. These pros and cons are summarized in Table 1 .

It is important to note that the aircraft used in the present work was designed according to a serial architecture, but the proposed method is applicable to both serial and parallel architectures. The authors would also like to note that these are not the only possible architectures, and several variants can be found in between the two mentioned architectures. Interested readers are kindly referred to specialized literature (Guzzella and Sciarretta, 2013).

\section{Hybrid-electric aircraft: the Panthera hybrid}

To test the novel proposed methods, the preliminary design data of an aircraft already under construction will be used. This aircraft is the hybrid version of the Panthera aircraft, manufactured by Pipistrel, known as Panthera hybrid. The original aircraft is a four-seat general aviation aircraft (Figure 2), and the hybrid version will use the same airframe, but the powertrain will be a hybrid-electric system with serial

\section{Figure 2 Panthera aircraft}

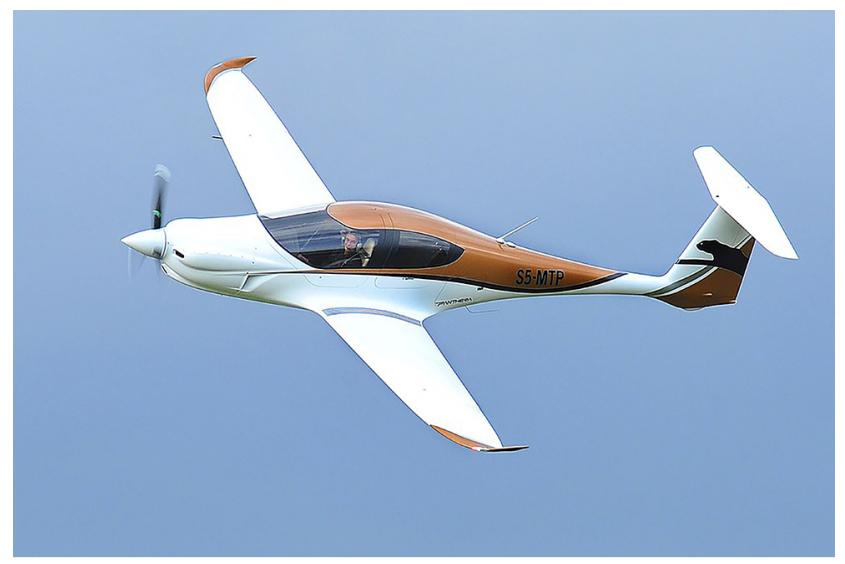

Source: Pipistrel d.o.o Ajdovščina

Table 1 Pros and cons of different hybrid electric architectures

\begin{tabular}{lll}
\hline & Pros & Cons \\
\hline Parallel & Redundancy can easily be achieved in some cases & Complex mechanical coupling mechanisms \\
Serial & Highly modular & Complex electrical systems \\
\hline
\end{tabular}


architecture, matching the one shown in Figure 1(a) (MAHEPA consortium, 2017a).

The Panthera hybrid is powered by an electric motor rated to $300 \mathrm{~kW}$ (150 kW continuous). The motor is powered by two electricity sources: Li-ion batteries and a generator powered by a turbocharged piston engine. For redundancy, both the electric motor and the generator are dual winding machines connected to dual inverters. The IC engine powering the generator provides up to $100 \mathrm{~kW}$ in continuous operation (MAHEPA consortium, 2017b).

The batteries of the aircraft are still under development thus approximate data was used in this study. The batteries are considered to store up to $20 \mathrm{kWh}$ of energy and weigh about $100 \mathrm{~kg}$. The maximum capacity of the fuel tanks is $210 \mathrm{~L}$. Based on the density of gasoline, one can conclude that the batteries store approximately $1 \%$ of the total energy onboard despite having approximately $63 \%$ of the total energy storage mass (fuel tank mass not taken into account).

\section{Problem: optimal onboard energy management}

As already described, having a hybrid aircraft creates a whole new range of problems to be investigated, especially in terms of energy control. In the case of conventional aircraft, there is only one source of energy, fuel, and the rate of fuel consumption is fully defined by the desired engine performance at a given instant (Roskam and Lan, 1997). That is not the case with hybrid aircraft, where more than one source of energy is available, and the rate of consumption of each source must be selected to yield the desired engine(s) performance. The selection of the energy consumption rates for an hybrid aircraft is thus an underdetermined problem, opening a new degree of freedom that can be used for optimization (Harmon et al., 2006; Bradley et al., 2009; Perullo and Mavris, 2014; Bongermino et al., 2017; Donateo et al., 2018b).

In most hybrid aircraft designs, it is assumed that there is an automatic controller selecting the amount of energy to be drained from each available source at each moment (i.e. in real time). This controller can be programmed in many ways and with many objectives such as improving take off performance or decreasing noise during approach. In the specific case under study, the controller has the objective of decreasing total fuel consumption and does this by selecting the throttle position of the IC engine powering the generator ("generator throttle"), while the pilot takes care of the electric motor driving the propeller (i.e. the "normal" throttle).

These automatic controllers envisioned for hybrid aircraft can have different levels of complexity and share several similarities with the ones used in hybrid cars, as they serve the same propose: onboard energy management. But, unlike cars, the trajectory of an aircraft is usually much better known, $a$ priori, i.e. before the trip actually begins. This opens the door for new types of high performance controllers, of types not generally used in the car industry.

The best possible controllers are called optimal controllers (Betts, 1998). In a comparison with conventional controllers, optimal controllers are computationally heavier and require an a priori knowledge of all the external disturbances applied to the system. The flight trajectory is usually defined before the flight and, thanks to the development of low cost and low-weight powerful computers, these issues became straightforward. Even in the case when optimal controllers are not used in real time during the mission, they are still of utmost importance; they are used to quantify the performance of a real time controller. This is regularly done when studying the performance of controllers for hybrid cars (Guzzella and Sciarretta, 2013; Sinoquet et al., 2009; Liu and Peng, 2008).

A major difference between optimizing hybrid cars and hybrid aircraft is the high correlation between weight and performance in the latter case. This effect is usually neglected when designing a controller for cars but becomes important when dealing with aircraft. Addressing this effect in energy management was only done in qualitative ways (Perullo and Mavris, 2014), and one of the objectives of this work is to address it in a quantitative way.

To conclude the remarks on the optimal energy management problem, all optimizations require an objective function (a target quantity to be minimized or maximized). As already noted, in this work, the objective is to minimize the total fuel consumption. Other metrics may be relevant for this problem, such as the minimization of total energy, minimization of total energy cost or more advanced metrics (Pornet et al., 2014).

\section{Optimal control for a hybrid aircraft}

In this section, the methodology used to tackle the problem of optimal energy management for hybrid aircraft will be detailed. Before starting the detailed explanation, the overall approach is presented.

\section{Methodology roadmap}

The problem under study is an optimization problem and, to perform the optimization itself, the DP method will be used. The reasons why this method was chosen will be detailed in the next sections. The aircraft will be modelled as a point-mass model, as the scope of this work is performance dynamics only. The trajectory/mission will be fixed (i.e. not subjected to optimization), making the required flight power at each instant of the mission completely defined. The optimizer will then optimize the power delivered by the IC engine during the mission to minimize fuel consumption.

The use of this simplified performance model coupled with an optimizer allows one to study what are the optimal "generator throttle" settings for such a mission. More importantly, it also allows studying which parameters of the mission and/or aircraft have the largest effects on the optimization results.

\section{Optimal control}

The problem one is trying to solve belongs to a class of problems usually called "optimal control problems". Several different solution methods have been applied to a variety of problems both in aeronautics and astronautics (Betts, 1998; Rao, 2009; Guzzella and Sciarretta, 2013).

The results presented in the present work were obtained using one of the oldest methods available: the DP method. This method was selected for two main reasons: it is easy and straightforward to use and, if properly implemented, always yields the global optimal solution for the problem. Unfortunately, the solution is optimal only if all the external disturbances applied to the system are known beforehand, 
being this method more useful for checking the performance of real-time controllers than for a real-life application. Besides, it tends to be quite computationally expensive.

\section{Dynamic programming and solver}

The DP method was originally introduced by Richard Bellman in the late 1950s (Bellman, 1957). Only a short description of the methodology will be given, and readers interested in details are referred to specialised texts (Bellman, 1957; Bertsekas, 2017; Guzzella and Sciarretta, 2013).

The base of DP is the so-called "principle of optimality" (Bellman, 1957): "Every part of an optimal control policy is an optimal control policy". For example, if a state trajectory from state $A$ to state $C$ is optimal and passes through state $B$, then the segment of the trajectory from B to C should also be optimal. Taking this into account, the optimal trajectories (and related control policies) can be calculated by going back in time: for every time step, there is an optimal trajectory that allows achieving the desired final state. Soon the costs for all the possible trajectories are known, the optimal trajectory starting from the initial state can be selected (and consequently the optimal control policy).

The DP solver used in this work is open source and was developed at the Institute for Dynamic Systems and Control ETH Zürich. The solver was introduced and validated in the literature (Sundstrom and Guzzella, 2009; Sundström et al., 2009; Elbert et al., 2013). In this work, the solution is found by modelling the aircraft as a mathematical model which is evaluated during small-time steps for given initial states, external conditions and controls. The output of the model is the final state at the end of a time step. By evaluating the outputs and managing the inputs, the model is continuously reevaluated until the optimal initial states and control policies to achieve a given final condition are fully determined.

\section{Dynamic programming for hybrid vehicle problems}

Among other techniques, DP is regularly used to quantify the performance of real time controllers in hybrid cars (Guzzella and Sciarretta, 2013; Sinoquet et al., 2009; Liu and Peng, 2008). This is owing to its "most wanted" characteristic of yielding the global optimum for the problem. The main problem with DP is the so-called "curse of dimensionality": the problem scales exponentially with the number of states and controls. This is usually not a problem for hybrid car problems, were there is usually just one state: the state of charge (SoC) of the battery.

When dealing with hybrid aircraft, the SoC can be used as solo state (Bongermino et al., 2017). However, to achieve more meaningful results, the weight of the vehicle must also be accounted as state. This is especially important in long-range missions, where the reduction of mass owing to fuel consumption greatly modifies the aircraft performance. The weight becomes then a second state, requiring a substantial increase in computation power and memory requirements when compared to a single state case.

Summarising, the two states of the system to be optimized are weight $(W)$ and SoC, and the corresponding equations of state are as follows:

$$
\begin{gathered}
\dot{W}=-\dot{m}_{f} \cdot g \\
S \dot{O} C= \begin{cases}-I_{b a t} / Q_{0} & \text { if } I_{\text {bat }} \geq 0 \\
-\eta_{c} \cdot I_{b a t} / Q_{0} & \text { if } I_{\text {bat }}<0\end{cases}
\end{gathered}
$$

The equations of state are integrated in time for a time step through a simple Euler integration, and the resulting states feed into the DP algorithm. Details on how $\dot{m}_{f}$ and $I_{b a t}$ are computed will be given in the next section.

It is important to stress that, against common practice in other aircraft performance studies, speed and altitude are not states, and subsequently, they are not subjected to optimization. Speed and altitude should instead be regarded as variable external inputs (see section Mission parameters). This is necessary to avoid the "curse of dimensionality" because an excessive number of states would make the simulations prohibitively expensive. In this study, the focus is on the optimization of the "internal works" of a hybrid aircraft, i.e. the internal energy management. Problems including more states are regarded as matter for future work.

\section{Modelling}

To simulate (and optimize) the trajectory of any vehicle or a figure of merit (e.g. fuel consumption) for a given trajectory, a mathematical model describing the dynamics of the vehicle is generally needed. This is also the case when DP is used as optimization method.

The aircraft performance is modelled using the classical point-mass model with some typical simplification: the angle of attack and thrust line angle are assumed to be small, and $C_{D}$ is assumed to be function of $C_{L}$ only (no Mach dependency, $C_{D}=$ $\left.C_{D}\left[C_{L}\right]\right)$. The whole system is regarded as quasi-static, which means that the transients of all the subsystems are neglected. In addition, the accelerations/decelerations to which the aircraft is subjected during climb and descent are ignored, but this approximation should be challenged in the future. Nevertheless, given the small and slow changes in speed during climb and descent, it is expected that the main findings of this work remain unchanged. The described simplifications are common in aircraft performance optimization studies (Roskam and Lan, 1997; Burrows, 1982).

Starting from the steady, symmetrical, powered flight equations of motion (Roskam and Lan, 1997):

$$
\begin{array}{r}
L-W \cos \gamma=0 \\
T-D-W \sin \gamma=0
\end{array}
$$

As at each time, step $W$ is known and $\gamma$ is predefined, $T$ can be computed. This allows to compute the flight power through the relation $P_{\text {flight }}=T \cdot V$

Another relevant approximation is the assumption that propeller, gearbox, motor, invertors and generator have constant efficiencies - a reasonable approximation in high-level preliminary studies like this work. It is interesting to note that, thanks to the electric drive and active control, it is possible to keep, for every flight condition, the propeller operating very close to its maximum efficiency point. Using this 
approximation, the flight conditions can be related with the electrical power flowing through the aircraft's electrical bus using the following expression:

$$
P_{\text {flight }}=\eta_{\text {prop }} \cdot \eta_{G B} \cdot \eta_{M} \cdot \eta_{i n v} \cdot\left(P_{b a t}+P_{\text {gen }}\right)
$$

The battery power $\left(P_{b a t}\right)$ relates with $I_{b a t}$ through the following:

$$
P_{b a t}=U_{b a t} \cdot I_{b a t}
$$

where $U_{b a t}$ is determined using a battery model. As $P_{\text {flight }}$ is determined by the flight conditions and weight and $P_{g e n}$ are defined by the control variable, $P_{b a t}$ and $I_{b a t}$ can then be computed for each time step.

The aircraft battery is a Li-ion cell pack with a $104 \mathrm{~S} 24 \mathrm{P}$ architecture. The modelling of the battery was done using two different implementations:

1 A standard implementation (Guzzella and Sciarretta, 2013), where the open-circuit voltage $\left(U_{b a t}\right)$ of the pack is regarded as, constant and only the ohmic losses and Coulombic efficiency are taken into account; this implementation is the one used for all the results presented throughout in this work.

2 A more complex modelling (Tremblay and Dessaint, 2009), where the open-circuit voltage depends on the SoC [1]; comparisons with the standard battery model for a reference trajectory indicated (negligible) differences on the final states of about $0.05 \%$.

All the cell characteristics are taken from Tremblay and Dessaint (2009), except the Coulombic efficiency. This parameter was assumed to be $95 \%$ and, because of this, subjected to a sensitivity study in the section Sensitivity studies. The maximum discharge rate of the battery is $15 \mathrm{C}$ owing to a requirement for an all-electric take off, but, in the present work, that is not simulated (see section Mission parameters for details). The maximum simulated discharge rate is thus much lower, $\sim 5 \mathrm{C}$ during climb. As the discharge C-rates are acceptable for this type of problems, the Peukert effect was not added to none of the battery models, although this approximation should be challenged in future work. Regarding charging, the battery recharge power is limited to $10 \mathrm{~kW}$ (or $0.5 \mathrm{C}$ ). All the propulsion system components are regarded as operating in a quasi-static regime.

Both the aircraft polar $\left(C_{D}=C_{D}\left[C_{L}\right]\right)$, and the efficiencies of the subsystems (among other relevant parameters) were obtained from the aircraft manufacturer, originating from flight tests or industrial experience, guaranteeing a reasonably high level of confidence and realism to the model, even after all the approximations mentioned previously.

\section{Engine modelling}

Special attention was devoted to the IC engine modelling. This was done following two different approaches.

In the first approach, the specific fuel consumption (SFC) is regarded as constant. This implies both generator power and fuel mass flow rate are linear functions of the generator throttle setting $\left(\Gamma_{f}=[0,100 \%]\right)$ which acts as sole control variable for the optimization:

$$
P_{\text {gen }}=P_{\text {gen }}\left(\Gamma_{f}\right)=\eta_{\text {gen }} \cdot P_{I C}, \quad \text { where } P_{I C}=P_{I C_{\max }} \cdot \Gamma_{f}
$$

$$
\dot{m}_{f}=\dot{m}_{f}\left(\Gamma_{f}\right)=S F C \cdot P_{I C}, \quad \text { where } P_{I C}=P_{I C_{\max }} \cdot \Gamma_{f}
$$

For the second approach, actual engine data obtained in bench tests was used. This data cannot be published in the present work, for confidentiality reasons. The bench tests were performed at two different atmospheric pressures to simulate

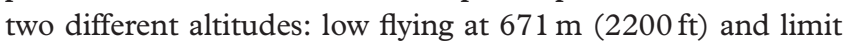
operating altitude of the engine (undisclosed, above $6,500 \mathrm{~m}$ ). Owing to the turbocharger, differences between the two data sets are negligible and, in this work, only the low-altitude data was used. The raw data from the bench tests was fitted with a 3rd degree polynomial (for ease of implementation), and the fuel mass flow rate becomes a non-linear function of engine power. Equation (5b) is thus replaced by equation (6):

$$
\dot{m}_{f}=\dot{m}_{f}\left(P_{I C}\right), \quad \text { where } P_{I C}=P_{I C_{\max }} \cdot \Gamma_{f}
$$

where $\Gamma_{f}$ acts again as the sole control variable.

Bench test data showed an almost-linear increase in fuel consumption with increasing power, hinting that the first modelling approach is not imprecise for this engine. As one of the objectives of the present work is to investigate the effects of the main factors influencing optimal energy management, it was decided to follow the first approach as main approach. This wittingly makes the problem more "artificial" but allows to better investigate and understand the different factors without introducing too much "real world" disruptions in the modelling. Nevertheless, the second approach was also implemented, and a comparison between results obtained by following the two different approaches is presented.

\section{Battery recharge during descent}

Another detail that was modelled to make the simulation more realistic was the use of the propeller to recharge the battery and, at the same time, act as a speed brake. This, to the authors' best knowledge, is a novelty in aircraft optimal energy management problems. The maximum amount of power that can be harvested from the flow was calculated using the wind power equation (Kalmikov, 2017):

$$
P_{\max }=1 / 2 \cdot \rho \cdot A \cdot V^{3} \cdot C_{P}
$$

where $\rho$ is the air density, $A$ is the rotor area, $V$ is the flow speed and $C_{P}$ is the rotor power coefficient.

A propeller is not designed to work as a windmill, so a conservative value for the power coefficient was selected $\left(C_{P}=\right.$ $0.3)$. This is comparable to an old farm windmill and quite below the typical values of $0.45-0.50$ for modern large-scale wind turbines (Hau, 2005).

As explained previously, this equation defines the maximum power that can be harvested by the rotor (= the propeller) for a given flow speed (= flight speed). $-P_{\max }$ is used as the lower limit for the (negative) propulsive power, or, in other words, the maximum amount of braking power that can be applied by the wind-milling propeller without loss of energy. 
Nevertheless, for most of the flight conditions, the practical recharge limit of the battery is above this limit, meaning that, in most of the situations, the propeller needs to operate at $C_{P}<$ 0.3 . In the modelling, it is assumed that the blade pitch angle of the propeller can be freely varied to regulate the rotating speed, and no operational limits are exceeded.

\section{Mission parameters}

To perform an optimization, a test mission had to be defined. This prescribed mission is representative of a typical mission performed by the Panthera hybrid.

The mission is set in terms of altitude, speed and flight path angle. As mentioned before, these parameters are fixed and are not regarded as states in the optimization problem, but cruise altitude and climb angle, being among the most important from an operational point of view, will be varied in the sensitivity studies (see section Sensitivity studies). This mission corresponds to a mission close to the maximum range of the aircraft without any use of reserve fuel. The fuel tanks are full at take-off, and $1 / 3$ of total fuel capacity is reserve fuel.

The aircraft takes-off at maximum take-off weight using only battery power, with the generator at idle for noise considerations. The generator only goes into full use above $305 \mathrm{~m}(1,000 \mathrm{ft})$ above ground. It was estimated that the energy corresponding to $\sim 20 \%$ of the battery charge is used for takeoff and initial climb, so the simulations start with battery at $80 \%$ and at $305 \mathrm{~m}(1,000 \mathrm{ft})$. The mission is terminated at the same altitude. The climb and descent are made with $\gamma \pm 4^{\circ}$ (respectively) and at constant equivalent airspeed. The runway is at sea level, and a still international standard atmosphere is assumed. For simplification, the transitions between flight phases (e.g. climb to cruise) are regarded as instantaneous. All the relevant mission parameters are plotted in Figure 3.

As a final remark, one should note that for each mission, a different optimization result will be obtained; although the control allocation result is optimal, it is only optimal for each individual mission.

\section{Figure 3 Prescribed mission}
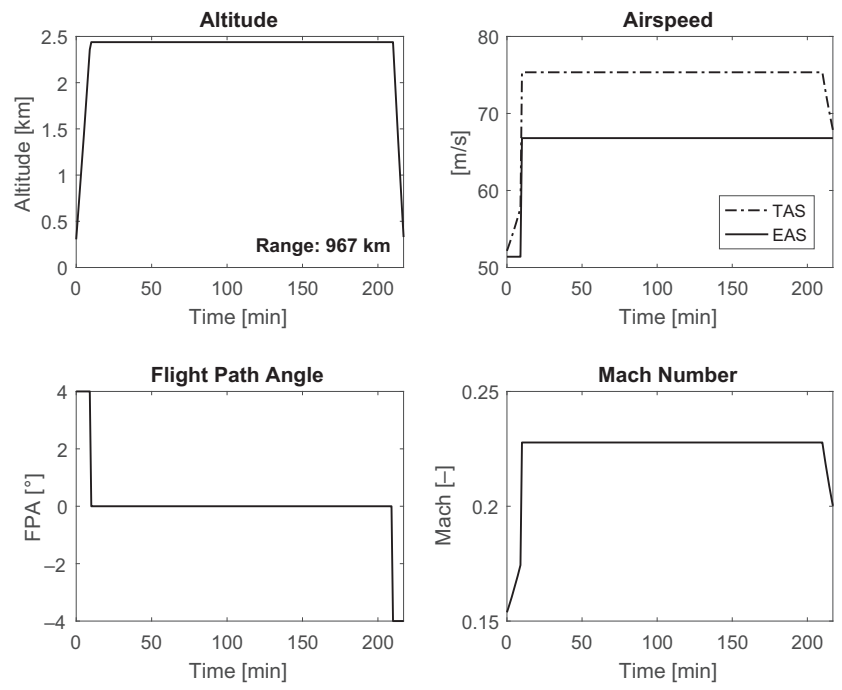

\section{Discretization}

DP requires states, controls and time to be discretized. The time step is $60 \mathrm{~s}$, fairly bellow the characteristic time of the dynamics under study. Both states (SoC and weight) are discretized in 121 discrete levels, while the single control ("generator throttle") is discretized in 101 discrete levels. The discrete levels are the possible values for the mentioned quantities between their minimum and maximum value. A mesh convergence study is presented in section Mesh convergence studies.

\section{Results}

The results of the optimization of energy management for the Panthera hybrid during a representative mission are presented in this section. As already stated, all optimizations were performed using DP and along the prescribed mission.

First, the basic optimal results for this mission are going to be presented. These results confirmed the expected behaviour previously described in the literature. Next, the optimal results will be compared with a non-optimal energy management, and conclusions will be drawn. Afterwards, the results obtained using the two modelling approaches for the IC engine will be compared. Finally the hybrid aircraft performance will be compared with the conventional aircraft, and results regarding the mesh convergence study will be presented.

Optimal energy management for the prescribed mission The optimized control and state trajectories, for the given mission, are synthesized in Figures 4 and 5. Two cases are presented: one in which the final battery SoC is free (Figure 4) and another in which the final battery SoC is constrained to 100\% (Figure 5).

One can easily confirm that the expected optimal use of battery stored energy is the one expected from reasoning (Perullo and Mavris, 2014): after the cruise altitude is reached, the battery is basically not used until the last part of the cruise or is recharged as soon as possible (depending on the final battery SoC constrain).

Figure 4 Optimal states and control for the given mission - free battery final state
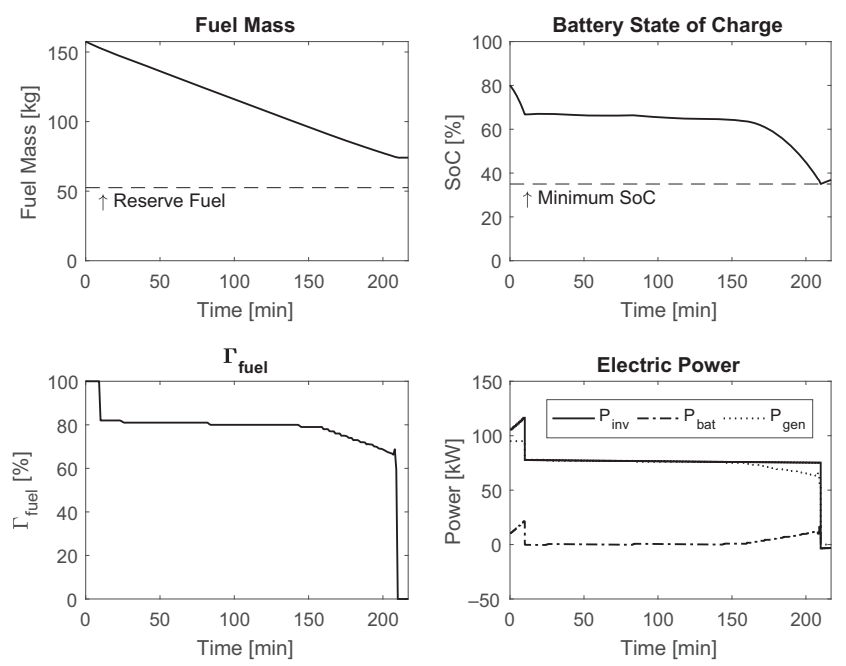
Figure 5 Optimal states and control for the given mission - full battery at the end of the mission
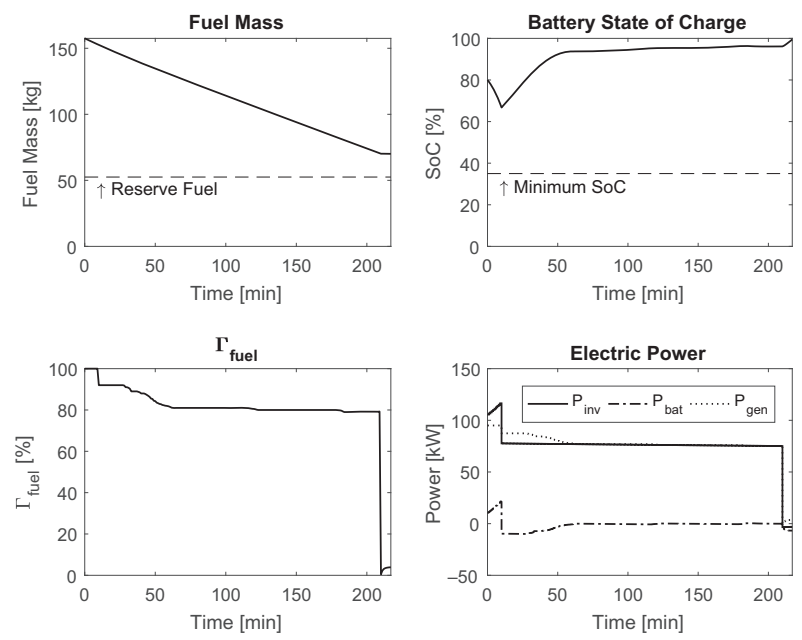

This "behaviour" is easy to understand: burning as much fuel as possible in the initial portion of the mission means that the aircraft will fly lighter for the remaining portion. Flying lighter means less propulsive power, making the aircraft more "efficient".

Regarding the computational effort needed to reach a solution, the DP solver takes about $100 \mathrm{~s}$ to calculate the solution for this problem in a conventional Windows workstation.

\section{Optimal vs non-optimal results}

To test the gain obtained with the optimization, the same mission was also "flown" using non-optimized controls. Three different cases are presented in Figure 6.

\section{“On-Off” Control}

A non-optimal solution: the engine/generator is kept at approximately $80 \%$ throttle for most of the flight. During descent, the engine is at idle.

Figure 6 Optimal vs non-optimal results
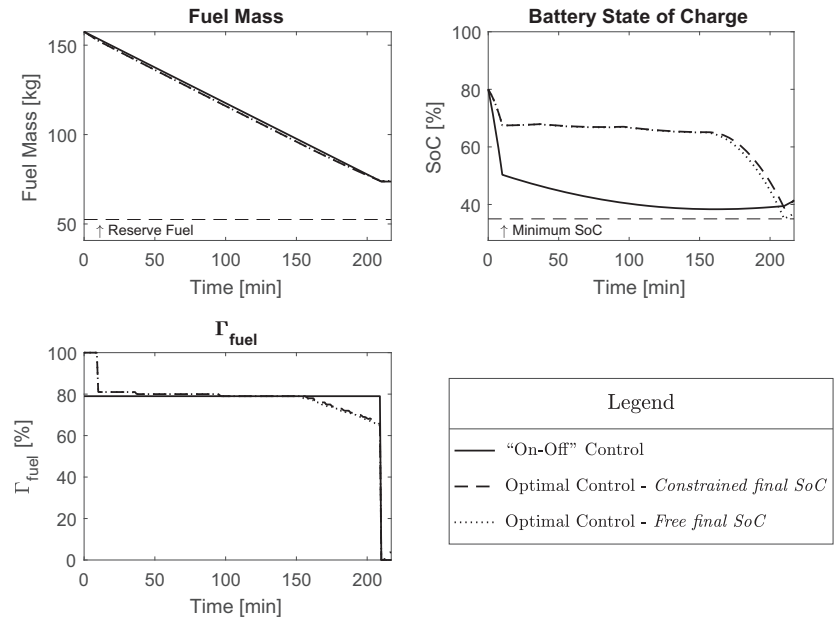

Optimal control - constrained final SoC

A solution with optimal control and the final battery SoC equal to the value in the "On-Off" case. The objective is to compare directly the fuel used between the two cases.

\section{Optimal control - free final SoC}

A solution with optimal control and no constrains on the battery, the final case, is presented also in Figure 4.

As one can observe, in the final fuel consumption, there are almost no differences. From the first to the second case, there is only an improvement of $0.06 \%$ in total fuel consumption, while from the first to the third case, the improvement is $0.4 \%$.

One very interesting conclusion can be drawn from these results is that the gains in optimizing the generator output are so marginal that it is more interesting, from an engineering point of view, to invest in using/developing an engine optimized for one single "set point" and thus eventually minimizing the weight of the system, fuel consumption and cooling requirements. In a nutshell, going from a "throttleble" engine logic to an auxiliary power unit logic with only "idle" or "on" states.

If this "On-Off" reasoning is carried on, an important conclusion is reached that the generator design and selection depends only on the desired cruise performance, i.e. the desired cruise speed. This happens only because the battery is "taking care" of the high demand power segments of the flight, namely, the take-off and initial climb. In other words, the two systems can be designed independently, and modularity is easy to implement.

It should also be noted that the small differences between the optimal and non-optimal cases are expected to increase if more energy is stored in the batteries with respect to the total energy onboard.

\section{Internal combustion engine - comparison of modelling approaches}

The two IC engine modelling approaches presented in section Engine modelling were compared to see the effect of a more realistic engine model on the optimal solution. By coincidence, the results of the second approach have some similitude with the "On-Off" control results presented in Figure 6. There is a simple explanation for this: the bench test data shows an increase in SFC for higher throttle settings $\left(\Gamma_{f}>80 \%\right)$, and the optimizer avoids that region of high consumption even during the highly demanding climb phase. And, unlike the nonoptimal solutions, the use of a realistic engine model yields improvements in the results; the fuel consumption is $0.94 \%$ lower, and the final SoC is $0.13 \%$ higher when compared to the constant SFC case (Figure 7).

These results seem to indicate that the optimal solution is very sensitive to system modelling and modelling uncertainties. Similar conclusions will be found on the section Sensitivity studies.

\section{Comparison with conventional propulsion}

Comparing the experimental hybrid Panthera with the conventionally powered Panthera is extremely difficult. The experimental aircraft has a payload different from the conventional one and several systems, including the propeller, are different. A direct comparison would never be fair 
Figure 7 Engine modelling comparison - constant SFC vs fitted bench test data
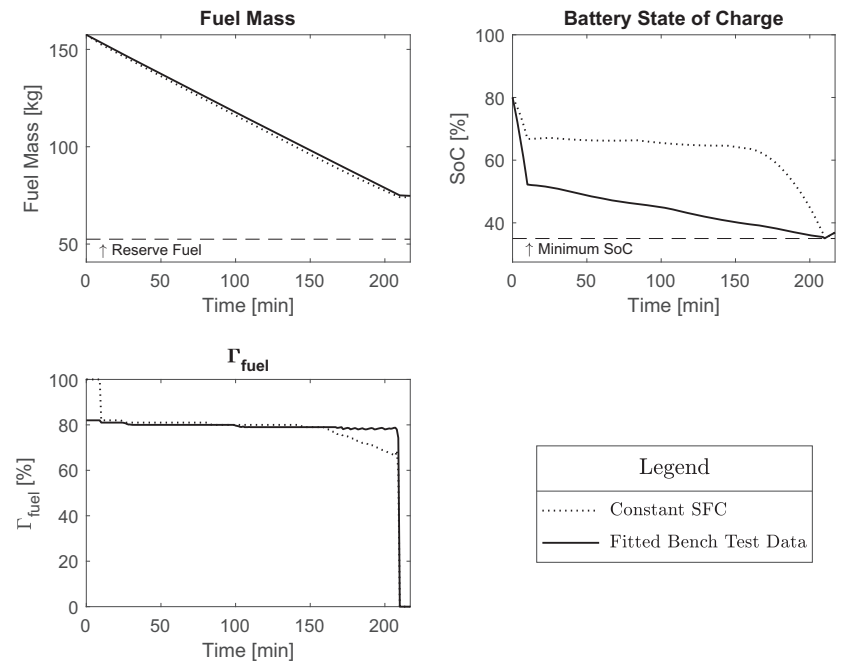

comparison. Keeping this in mind, some results from previous work (MAHEPA consortium, 2017b) will be presented for the reader's information:

- Regarding cruise flight, the engine powering the generator in the hybrid Panthera would have fuel consumption $1.8 \%$ lower than the engine of the conventional Panthera. These values are for the power required to sustain a cruise at $8,000 \mathrm{ft}(2,438 \mathrm{~m})$ and $158 \mathrm{kn}(81 \mathrm{~m} / \mathrm{s})$.

- Regarding climb flight, the hybrid Panthera would consume $22 \%$ less fuel to perform a standard climb, when compared with its conventional counterpart. Note that, as stated before, only battery power is used during take-off and initial climb thus also minimizing noise pollution.

\section{Mesh convergence studies}

To verify the numerical quality of the results, a mesh convergence study was performed. The number of discretization steps was increased to 605 for the weight state and 242 for the SoC state. The control parameter discretization was increased to 303 steps.

Differences on the final states $0.03 \%$ were observed, together with no significant differences in the control history or state trajectories. This is considered to be a good indication of the numerical validity of the results.

\section{Sensitivity studies}

Several parameters were tested to determine how sensitive the final result was to them. Among the parameters, there was only one that showed significant effects in the final results: battery charging efficiency.

Battery charging efficiency, also called Coulombic efficiency, is a modelling parameter used to take into account the fact that not all the energy sent into the battery is converted back to electric charge (Guzzella and Sciarretta, 2013). This factor has several definitions and may or may not include the effects of the ohmic losses or other effects. This factor also depends on the battery chemistry, configuration or age. In the battery models used in this work, the ohmic losses are explicitly taken into account separately, and the value for the efficiency was taken from literature (Toman et al., 2016; Yang et al., 2018) as a way of compensating for the secondary above-mentioned effects. Values in the literature range from $90 \%$ to above $99 \%$ thus some exploration on this parameter was performed.

One can see the results in Figure 8, where all the state trajectories and controls are optimal. Despite the fuel consumption not changing meaningfully with different recharging efficiencies, it is clear that the control parameter (the "generator throttle") and the trajectory of the SoC change considerably depending on the efficiency value in the model.

Also in Figure 8, one can observe the effect of the battery recharge limit $(10 \mathrm{~kW})$ on the optimal control history: observe the differences on the two optimal controls for $\eta_{c}=0.99$ around $15 \mathrm{~min}$ into the flight.

Besides the battery charging efficiency, the sensitivity to variations in cruise altitude and climb angle was tested. For relevant operational conditions, the control policy and the corresponding trajectories do not change meaningfully (i.e. the "shape" of the control policy does not change). The only relevant difference is the SoC at the end of climb: higher altitude and/or higher climb angle lead to a lower SoC at the end of climb with respect to the baseline mission. The SoC plateau that follows the end of climb is also lower in these cases, with no evolution on the SoC level until the last phase of the mission (like in the baseline). These results are result of the same control policy for the climb seen before in the baseline case: IC engine at $100 \%$ power and the batteries supplying the needed extra power. Longer and/or stepper the climb, more energy is drained from the batteries and lower the SoC at the end of climb. This highlights the importance of the climb phase for the battery design.

\section{Conclusions}

In this work, a method to optimize the energy management for a hybrid-electric aircraft is presented. The method can be used during conceptual and preliminary design phases and uses a DP open source solver together with an aircraft performance

Figure 8 Effect of the battery recharge efficiency in the SoC trajectory
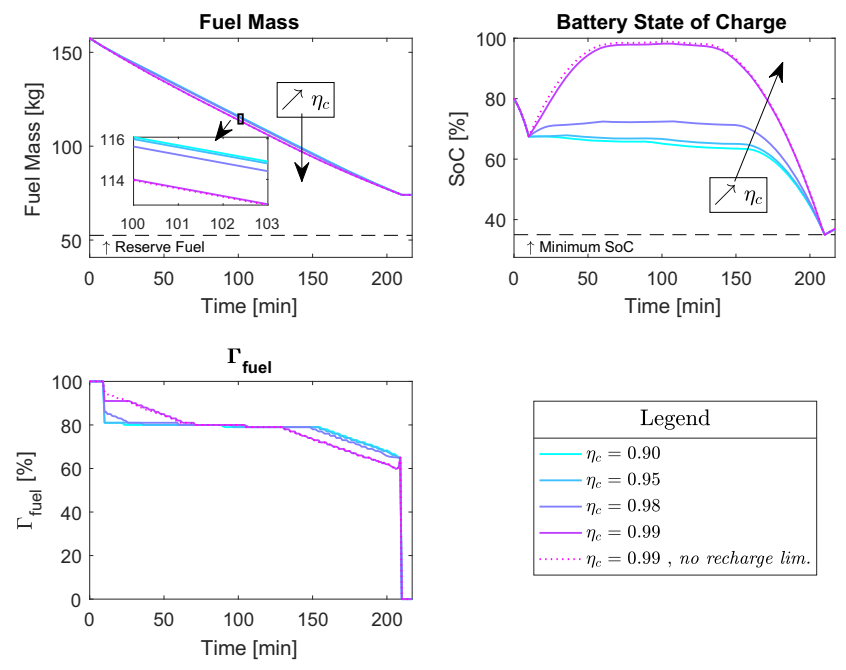
model. This model includes effects such as weight variations and in-flight energy recovery during the descent. It was shown that optimal controls only yield small improvements when compared with non-optimal results. A sensitivity study showed that the battery recharge efficiency can have a large impact in the control history despite having a small effect in the final fuel consumption (when optimal solutions are compared).

Regarding shortcomings of the presented method, it is important to summarize that it is only applicable "as-is" during the design and development phases of the aircraft. To use such a methodology in operational environments, it should be complemented by alternative methods, such as optimal control solvers robust to external disturbances.

\section{Future work}

The main recommended follow up for this work is to test the results against a different type of optimal control solver. This will allow to further test the validity of the results but will also allow the use of more complex aircraft models. Aircraft models with more states (e.g. speed or altitude) will give new insights on optimal trajectories and energy management, but models with extra states are difficult to study using DP owing to computational limitations. The author suggests the use of direct methods, namely, direct multiple shooting and/or collocation methods for further developments. The integration of the tool within conceptual aircraft design tools is also envisioned.

\section{Acknowledgements}

The authors gratefully acknowledge the support provided by the members of the MAHEPA consortium, especially Dr F. Oliviero and Pipistrel, for providing the aircraft data. The authors wish to give special thanks to the TU Delft members of the NOVAIR project for the fruitful discussions during the preparation of this work.

This research was partially funded by the Aerospace Engineering Faculty of TU Delft (AWEP department). This research was also partially funded by the MAHEPA project. The MAHEPA project has received funding from the European Union's Horizon 2020 research and innovation programme under the grant agreement No. 723368. This paper reflects only the authors' views, and the Union is not liable for any use that may be made of the information contained therein.

\section{Note}

1. But neglecting the polarization resistance because of implementation limitations.

\section{References}

Bellman, R. (1957), Dynamic Programming, Princeton University Press, Princeton, NJ.

Bertsekas, D.P. (2017), Dynamic Programming and Optimal Control, Vol. 1, Athena Scientific.
Betts, J.T. (1998), "Survey of numerical methods for trajectory optimization", Fournal of Guidance, Control, and Dynamics, Vol. 21 No. 2, pp. 193-207.

Bongermino, E., Mastrorocco, F., Tomaselli, M., Monopoli, V.G. and Naso, D. (2017), "Model and energy management system for a parallel hybrid electric unmanned aerial vehicle", 2017 IEEE 26th International Symposium on Industrial Electronics (ISIE), IEEE.

Bradley, T., Moffitt, B., Parekh, D., Fuller, T. and Mavris, D. (2009), "Energy management for fuel cell powered hybridelectric aircraft", 7th International Energy Conversion Engineering Conference, American Institute of Aeronautics and Astronautics.

Brelje, B. and Martins, J. (2018), "Electric, hybrid, and turboelectric fixed-wing aircraft: a review of concepts, models, and design approaches", Progress in Aerospace Sciences, Vol. 104.

Burrows, J.W. (1982), "Fuel optimal trajectory computation", Fournal of Aircraft, Vol. 19 No. 4, pp. 324-329.

Donateo, T., Ficarella, A. and Spedicato, L. (2018a), "Applying dynamic programming algorithms to the energy management of hybrid electric aircraft", Coal, Biomass, and Alternative Fuels; Cycle Innovations; Electric Power; Industrial and Cogeneration; Organic Rankine Cycle Power Systems, Vol. 3, American Society of Mechanical Engineers.

Donateo, T., Ficarella, A. and Spedicato, L. (2018b), "A method to analyze and optimize hybrid electric architectures applied to unmanned aerial vehicles”, Aircraft Engineering and Aerospace Technology, Vol. 90 No. 5.

Donateo, T., Pascalis, C.L.D. and Ficarella, A. (2019), "Synergy effects in electric and hybrid electric aircraft", Aerospace, Vol. 6 No. 3, p. 32.

Elbert, P., Ebbesen, S. and Guzzella, L. (2013), "Implementation of dynamic programming for ndimensional optimal control problems with final state constraints", IEEE Transactions on Control Systems Technology, Vol. 21 No. 3, pp. 924-931.

Ficarella, A., Pascalis, C.L.D. and Donateo, T. (2018), "Exploiting the synergy between aircraft architecture and electric power system in unmanned aerial vehicle through many-objective optimisation", International fournal of Sustainable Aviation, Vol. 4 Nos 3/4, p. 247.

Gohardani, A.S., Doulgeris, G. and Singh, R. (2011), "Challenges of future aircraft propulsion: a review of distributed propulsion technology and its potential application for the all electric commercial aircraft", Progress in Aerospace Sciences, Vol. 47 No. 5, pp. 369-391.

Guzzella, L. and Sciarretta, A. (2013), Vehicle Propulsion Systems, SpringerLink: Bücher, Springer Berlin Heidelberg.

Harmon, F.G., Frank, A.A. and Chattot, J.-J. (2006), "Conceptual design and simulation of a small hybrid-electric unmanned aerial vehicle”, fournal of Aircraft, Vol. 43 No. 5, pp. 1490-1498.

Hau, E. (2005), Wind Turbines, 2nd ed., Springer, Berlin Heidelberg.

Hepperle, M. (2012), Electric Flight-Potential and Limitations, German Aerospace Centre, Institute of Aerodynamics and Flow Technology. 
Kalmikov, A. (2017), "Wind power fundamentals", Wind Energy Engineering, Elsevier, pp. 17-24.

Liu, J. and Peng, H. (2008), "Modeling and control of a powersplit hybrid vehicle", IEEE Transactions on Control Systems Technology, Vol. 16 No. 6, pp. 1242-1251.

MAHEPA consortium (2017a), D1.1: Concept of Modular Architecture for Hybrid Electric Propulsion of Aircraft, Technical report, MAHEPA project.

MAHEPA consortium (2017b), D2.1 Performance and Energy Efficiency Trade-off Study, Internal Document - Private Communication, MAHEPA project.

Mavris, D.N., Chakraborty, I., Garcia, E., Perullo, C.A. and Trawick, D.R. (2010), "Onboard energy management", Encyclopedia of Aerospace Engineering, pp. 1-10.

Perullo, C. and Mavris, D. (2014), "A review of hybrid-electric energy management and its inclusion in vehicle sizing", Aircraft Engineering and Aerospace Technology, Vol. 86 No. 6, pp. 550-557.

Perullo, C.A., Trawick, D., Clifton, W., Tai, J.C.M. and Mavris, D.N. (2014), "Development of a suite of hybrid electric propulsion modeling elements using NPSS", Aircraft Engine; Fans and Blowers, Vol. 1A, American Society of Mechanical Engineers.

Pornet, C. and Isikveren, A. (2015), "Conceptual design of hybrid-electric transport aircraft", Progress in Aerospace Sciences, Vol. 79, pp. 114-135.

Pornet, C., Kaiser, S. and Gologan, C. (2014), "Cost-based flight technique optimization for hybrid energy aircraft", Aircraft Engineering and Aerospace Technology, Vol. 86 No. 6, pp. 591-598.

Pornet, C., Gologan, C., Vratny, P.C., Seitz, A., Schmitz, O., Isikveren, A.T. and Hornung, M. (2013), "Methodology for sizing and performance assessment of hybrid energy aircraft", 2013 Aviation Technology, Integration, and Operations Conference, American Institute of Aeronautics and Astronautics.

Rao, A.V. (2009), "A survey of numerical methods for optimal control", Advances in the Astronautical Sciences, Vol. 135 No. 1, pp. 497-528.

Rosero, J.A., Ortega, J.A., Aldabas, E. and Romeral, L. (2007), "Moving towards a more electric aircraft", IEEE Aerospace and Electronic Systems Magazine, Vol. 22 No. 3, pp. 3-9.

Roskam, J. and Lan, C.-T.E. (1997), Airplane Aerodynamics and Performance, DARcorporation.

Sarlioglu, B. and Morris, C.T. (2015), "More electric aircraft: review, challenges, and opportunities for commercial transport aircraft", IEEE Transactions on Transportation Electrification, Vol. 1 No. 1, pp. 54-64.

Sehra, A.K. and Whitlow, W. (2004), "Propulsion and power for 21 st century aviation", Progress in Aerospace Sciences, Vol. 40 Nos 4/5, pp. 199-235.

Serrao, L., Onori, S. and Rizzoni, G. (2011), “A comparative analysis of energy management strategies for hybrid electric vehicles", Fournal of Dynamic Systems, Measurement, and Control, Vol. 133 No. 3.

Silvas, E., Hofman, T., Murgovski, N., Etman, P. and Steinbuch, M. (2016), "Review of optimization strategies for system-level design in hybrid electric vehicles", IEEE Transactions on Vehicular Technology, pp. 1-1.

Sinoquet, D., Rousseau, G. and Milhau, Y. (2009), "Design optimization and optimal control for hybrid vehicles", Optimization and Engineering, Vol. 12 Nos 1/2, pp. 199-213.

Sundstrom, O. and Guzzella, L. (2009), "A generic dynamic programming matlab function”, 2009 IEEE International Conference on Control Applications, IEEE.

Sundström, O., Ambühl, D. and Guzzella, L. (2009), “On implementation of dynamic programming for optimal control problems with final state constraints", Oil $\mathcal{E}$ Gas Science and Technology - Revue de L'Institut Français du Pétrole, Vol. 65 No. 1, pp. 91-102.

Toman, M., Cipin, R., Cervinka, D., Vorel, P. and Prochazka, P. (2016), "Li-ion battery charging efficiency", ECS Transactions, Vol. 74 No. 1, pp. 37-43.

Tremblay, O. and Dessaint, L.-A. (2009), "Experimental validation of a battery dynamic model for EV applications", World Electric Vehicle fournal, Vol. 3 No. 2, pp. 289-298.

Voskuill, M., van Bogaert, J. and Rao, A.G. (2017), "Analysis and design of hybrid electric regional turboprop aircraft", CEAS Aeronautical fournal, Vol. 9 No. 1, pp. 15-25.

Wall, T.J. and Meyer, R. (2017), "A survey of hybrid electric propulsion for aircraft", 53rd AIAA/SAE/ASEE foint Propulsion Conference, American Institute of Aeronautics and Astronautics.

Yang, F., Wang, D., Zhao, Y., Tsui, K.-L. and Bae, S.J. (2018), "A study of the relationship between coulombic efficiency and capacity degradation of commercial lithiumion batteries", Energy, Vol. 145, pp. 486-495.

\section{Corresponding author}

José Pedro Soares Pinto Leite can be contacted at: jpsp. leite@gmail.com 